\title{
Erratum to: Quantifying the health benefits of chronic disease prevention: a fresh approach using cardiovascular disease as an example
}

\author{
Nicholas J. Wald ${ }^{1}$ Joan K. Morris ${ }^{1}$
}

Published online: 27 May 2015

(C) Springer Science+Business Media Dordrecht 2015

\section{Erratum to: Eur J Epidemiol (2014) 29:605-612 DOI 10.1007/s10654-014-9932-1}

Computing errors in our paper introduced small errors. We here provide the corrected estimates together with correct versions of the six tables (Tables $1,2,3,5,6,7$ ) that contained errors (available on the journal website linked to this paper). The authors apologize for the inconvenience caused by the following errors.

\begin{abstract}
Under the holistic model, $34 \%$ (not $33 \%$ ) of people who take the polypill from age 50 benefit, gaining on average 7.7 (not 8.0) years of life without an MI or stroke (18\% benefit under the reductionist model-not $19 \%)$. Estimates for reducing salt intake by $6 \mathrm{~g} /$ day are $34 \%$ and 2.6 years, respectively, (not $33 \%$ and 2.8 years) under the holistic model and $5 \%$ (not $6 \%$ ) under the reductionist model.
\end{abstract}

The online version of the original article can be found under doi:10.1007/s10654-014-9932-1.

Nicholas J. Wald

n.j.wald@qmul.ac.uk

1 Wolfson Institute of Preventive Medicine, Barts and The London School of Medicine and Dentistry, Queen Mary University of London, Charterhouse Square,

London EC1M 6BQ, UK

\section{Results}

Para 1: $56 \%$ should read $53 \% .0 .37 \%$ should read $0.53 \%$. $33 \%$ should read $34 \%$. 8.0 years should read 7.7 years. $67 \%$ should read $66 \%$.

Para 2: $33 \%$ should read $34 \%, 8.0$ years should read 7.7 years.

Para 3: $33 \%$ should read $34 \%$.

Para 4: $33 \%$ should read $34 \%, 2.8$ years should read 2.6 years and 8.8 years should read 8.7 years.

\section{Discussion}

Para 2: $19 \%$ should read $18 \%, 33 \%$ should read $34 \%$, $15 \%$ should read $16 \%, 8.0$ should read 7.7 .

Para 3: $33 \%$ should read 34\%, 2.8 years should read 2.6 years, $6 \%$ should read $5 \%$.

Para 4: $81 \%$ should read $78 \%, 55 \%$ should read $54 \%$, $1.0 \%$ should read $1.33 \%$.

Para 5: $33 \%$ should read $34 \%, 50 \%$ should read $46 \%$.

\section{Appendix}

Section 2: Estimating the age specific relative risk of a myocardial infarction or stroke on the Polypill.

Para 2: $56 \%$ should read $53 \%, 81 \%$ should read $78 \%$. 
Table 1 Estimates relating to the prevention of a first myocardial infarction (MI) or stroke ("disorder") in people taking polypill from specified ages

\begin{tabular}{lllllll}
\hline $\begin{array}{l}\text { Taking } \\
\text { polypill from } \\
\text { specified age } \\
\text { to age } 99\end{array}$ & $\begin{array}{l}\text { Proportion of people who } \\
\text { will have first MI or } \\
\text { stroke in the absence of } \\
\text { treatment (\%) }\end{array}$ & $\begin{array}{l}\text { Proportion of people } \\
\text { who will have first MI } \\
\text { or stroke while taking } \\
\text { polypill }(\%)\end{array}$ & $\begin{array}{l}\text { Relative risk } \\
\text { reduction }(\%)\end{array}$ & $\begin{array}{l}\text { Absolute } \\
\text { annual } \\
\text { risk } \\
\text { reduction } \\
(\%)\end{array}$ & $\begin{array}{l}\text { Proportion } \\
\text { of people } \\
\text { who benefit } \\
(\text { HBp) }\end{array}$ & $\begin{array}{l}\text { Among those who } \\
\text { benefit: Average years of } \\
\text { life gained without an MI } \\
\text { or stroke (HBag) }\end{array}$ \\
\hline 50 & 34 & 16 & 53 & 0.53 & 34 & 7.7 \\
60 & 33 & 16 & 52 & 0.69 & 33 & 6.5 \\
70 & 32 & 16 & 51 & 0.95 & 32 & 5.1 \\
80 & 29 & 15 & 49 & 1.41 & 29 & 3.4 \\
\hline
\end{tabular}

Table 2 Estimates relating to the prevention of a first myocardial infarction (MI) or stroke in individuals aged 50 and above according to specified daily salt reduction

\begin{tabular}{lllllll}
\hline $\begin{array}{l}\text { Salt } \\
\text { reduction } \\
(\mathrm{g} / \text { day })\end{array}$ & $\begin{array}{l}\text { Proportion of people who } \\
\text { will have first MI or stroke } \\
\text { in the absence of treatment } \\
(\%)\end{array}$ & $\begin{array}{l}\text { Proportion of people who } \\
\text { will have first MI or stroke } \\
\text { with a reduced salt intake } \\
(\%)\end{array}$ & $\begin{array}{l}\text { Relative } \\
\text { risk } \\
\text { reduction } \\
(\%)\end{array}$ & $\begin{array}{l}\text { Absolute } \\
\text { annual risk } \\
\text { reduction } \\
(\%)\end{array}$ & $\begin{array}{l}\text { Proportion of } \\
\text { people who } \\
\text { benefit (HBp) } \\
(\%)\end{array}$ & $\begin{array}{l}\text { Among those who benefit: } \\
\text { average years of life gained } \\
\text { without an MI or stroke } \\
(\mathrm{HB} \text { ag })\end{array}$ \\
\hline 1.5 & 34 & 32 & 4 & 0.04 & 34 & 0.7 \\
3.0 & 34 & 31 & 8 & 0.09 & 34 & 1.4 \\
4.5 & 34 & 30 & 12 & 0.13 & 34 & 2.0 \\
6.0 & 34 & 28 & 16 & 0.17 & 34 & 2.6 \\
\hline
\end{tabular}

Table 3 The two measures of health benefit in people aged 50 and over according to different preventive interventions to reduce the risk of a first myocardial infarction (MI) or stroke

\begin{tabular}{llllll}
\hline & $\begin{array}{l}\text { Reducing } \\
\text { salt by } \\
6 \text { g/day }\end{array}$ & $\begin{array}{l}\text { Taking } \\
\text { simvastatin } \\
20 \mathrm{mg} \text { daily from } \\
\text { age 50 }\end{array}$ & $\begin{array}{l}\text { Taking three blood- } \\
\text { pressure-lowering drugs } \\
\text { at half standard dose } \\
\text { daily from age 50 }\end{array}$ & $\begin{array}{l}\text { Taking polypill } \\
\text { daily from age 50 } \\
\text { (all four drugs) }\end{array}$ & $\begin{array}{l}\text { Reducing salt by 6 g/day } \\
\text { and taking polypill daily } \\
\text { from age 50 }\end{array}$ \\
\hline $\begin{array}{l}\text { Proportion who benefit }\left(\mathrm{HB}_{\mathrm{p}}\right) \\
\begin{array}{l}\text { Among these: average years of } \\
\text { life gained without an MI or } \\
\text { stroke }\left(\mathrm{HB}_{\mathrm{ag}}\right)\end{array}\end{array}$ & $34 \%$ & $34 \%$ & $34 \%$ & $34 \%$ & $34 \%$ \\
\hline
\end{tabular}

Table 5 Age-specific relative risk estimates

\begin{tabular}{lll}
\hline $\begin{array}{l}\text { Age taking } \\
\text { polypill }\end{array}$ & $\begin{array}{l}\text { Relative risk of a first } \\
\text { stroke on daily polypill }\end{array}$ & $\begin{array}{l}\text { Relative risk of a first } \\
\text { myocardial infarction } \\
\text { on daily polypill }\end{array}$ \\
\hline 50 & 0.26 & 0.13 \\
60 & 0.28 & 0.23 \\
70 & 0.34 & 0.32 \\
80 & 0.44 & 0.36 \\
$90+$ & 0.51 & 0.38 \\
\hline
\end{tabular}

a Polypill contained amlodipine $2.5 \mathrm{mg}$, losartan $25 \mathrm{mg}$, hydrochlorothiazide $12.5 \mathrm{mg}$ and simvastatin $20 \mathrm{mg}$
Table 6 Average relative risk reductions (\%) of a first myocardial infarction or stroke according to age at starting polypill and years of follow-up

\begin{tabular}{lllll}
\hline \multirow{2}{*}{$\begin{array}{l}\text { Years } \\
\text { of follow-up }\end{array}$} & \multicolumn{4}{l}{ Age starting to take polypill daily } \\
\cline { 2 - 5 } & 50 & 60 & 70 & 80 \\
\hline 10 & 78 & 71 & 63 & 54 \\
20 & 73 & 64 & 55 & 49 \\
30 & 66 & 57 & 51 & - \\
40 & 58 & 52 & - & - \\
50 & 53 & - & - & - \\
\hline
\end{tabular}




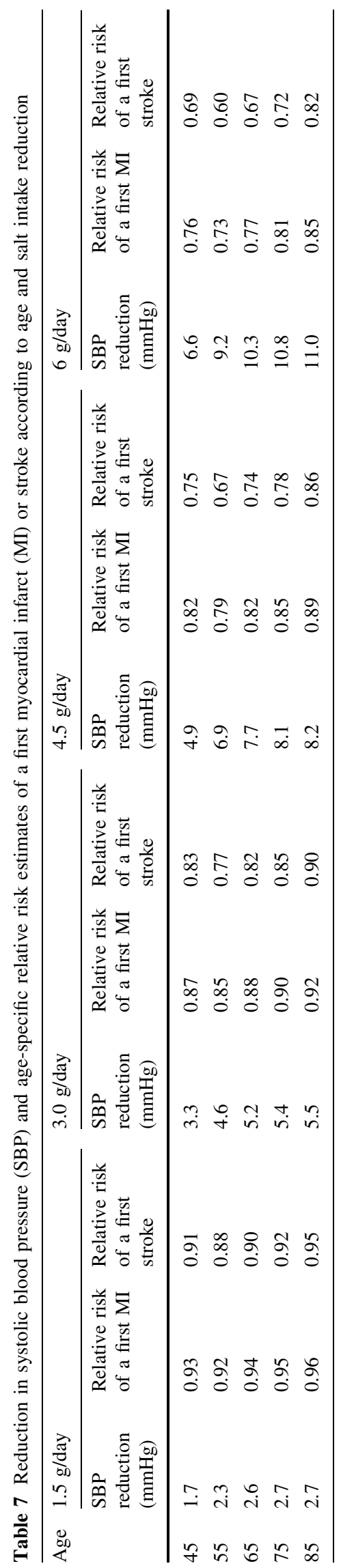

